\title{
Study on Nonuniform Deformation of Tailor Rolled Blank During Uniaxial Tension
}

\author{
Hua-Wei Zhang ${ }^{1} \cdot$ Xiang-Hua Liu ${ }^{2} \cdot$ Li-Zhong Liu ${ }^{3} \cdot$ Ping $\mathrm{Hu}^{3} \cdot$ Jia-Lu Wu ${ }^{1}$
}

Received: 16 November 2014/Revised: 2 July 2015/Published online: 12 September 2015

(C) The Chinese Society for Metals and Springer-Verlag Berlin Heidelberg 2015

\begin{abstract}
The deformation characteristics of tailor rolled blank (TRB) in the course of uniaxial tension were studied by means of analysis, test and simulation. The mechanical analytical model of TRB during uniaxial tension was set up, and the deformation formulae for the thinner side and for the thicker side were derived to quantify the deformation of TRB. On this basis, uniaxial tension tests on TRB and ordinary blanks (the thinner side and the thicker side of TRB) were conducted. Lagrange polynomial interpolation method was adopted to construct the stress-strain fields of unannealed and annealed TRBs for solving TRB material parameters, and then, uniaxial tension simulation on TRB was completed. Deformations and properties of unannealed TRB were compared with those of annealed TRB, and the thinner side and the thicker side were also compared. Finally, the research results were explained by metallurgical structure. The results show that nonuniform deformation happens in TRB during uniaxial tension, and the necking occurs on the thinner side. The agreement of analysis, test and simulation confirms the correctness of the analytical model and the deformation formulae. The findings of this paper can lay the foundation for the future study on TRB stamping formability and provide a way for TRB modeling.
\end{abstract}

\section{KEY WORDS: Tailor rolled blank (TRB); Nonuniform deformation; Uniaxial tension; Annealing;} Metallographical

\section{Introduction}

Tailor rolled blank (TRB) is another kind of lightweight metal sheet based on a new material processing technique after tailor welded blank (TWB) [1,2]. TRB is much better

Available online at http://link.springer.com/journal/40195

Hua-Wei Zhang

zhanghw@neuq.edu.cn

1 School of Mechanical Engineering and Automation, Northeastern University, Shenyang 110819, China

2 Research Institute of Science and Technology, Northeastern University, Shenyang 110819, China

3 School of Automotive Engineering, Dalian University of Technology, Dalian 116024, China than TWB in terms of mechanical properties, weight-saving effect, surface quality and production cost [3]. TRB demonstrates great potential and bright future, and TRB can play a great role in the fields of automobile and aerospace by substituting TWB with the same material, the same width and the different thicknesses [4].

In the production of stamping parts, the shape of parts will exert great influence on the formality of metal sheet, while the mechanical properties are crucial to the part quality [5]. As a simple and practical method to test material properties, uniaxial tension test is widely used to evaluate the basic mechanical properties of material.

Stress-strain relation under static loads as well as the basic mechanical parameters can be obtained by uniaxial tension test. These performance indexes are not only the calculation basis for engineering application and scientific research, but also the main evidence for material evaluation 
and selection as well as processing technique selection. Since the thickness of TRB changes in the rolling direction, its mechanical properties, different from an ordinary blank, require further study [6].

The deformation behavior of TWB has been studied by using analytical models, and the effects of process parameters and geometric parameters on tensile properties of TWB have been analyzed [7-10]. Some valuable conclusions were drawn from researches above, which proves the necessity for studying the material behaviors in tension for metal sheets with variable cross section. Li et al. [11] discussed the influence of thickness ratio and transition zone length of st16 TRB on the elongation rate and the movement of transition zone. Bao [12] studied the mechanical properties of $\mathrm{X} 5 \mathrm{CrNi} 1810$ austenitic stainless steel TRB before and after solution treatment. Material deformation in the transition zone of TRB will affect the formability of the whole TRB and the quality of TRB parts, but there is no research on this aspect, and what is more, study on the deformation theory of TRB depicting the deformation behaviors of TRB is barely referred to. Therefore, it is necessary to analyze the deformation process of TRB and construct the deformation theory of TRB, which can precisely describe the deformation behaviors of TRB.

In this work, the basic mechanical properties of TRB are discussed through study on the uniaxial tension process of TRB. A mechanical analytical model for TRB uniaxial tension is set up, and the deformation formulae are derived. The deformation course of TRB is discussed, and the deformation of TRB is analyzed. The setup of deformation theory for TRB can describe the deformation behaviors of TRB quantitatively. On this basis, the stress-strain fields of unannealed and annealed TRBs are constructed, and then, tension test and simulation on TRB are performed to verify the correctness of the model and the formulae. Effects of annealing on deformation and properties of TRB are considered, and finally, the research results are explained by metallurgical structure. The research findings can not only provide theoretical foundations for analyzing the formability of TRB, but also present material input for the transition zone, which can decide whether FEA results about TRB formability are convincing or not.

\section{Mechanical Analysis of TRB During Uniaxial Tension}

\subsection{Mechanical Analytical Model}

Figure 1 shows the mechanical analytical model of TRB during uniaxial tension. That every part of TRB keeps the same width in the deformation process is presumed.

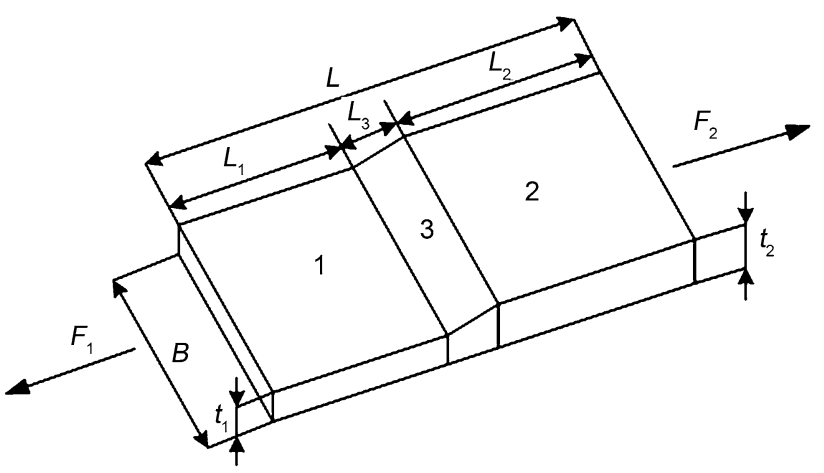

Fig. 1 Mechanical analytical model of uniaxial tension for TRB

According to the force equilibrium relation of TRB in the process of uniaxial tension, combined with exponential constitutive relation $\sigma=K \varepsilon^{n}$, the following equations can be obtained:

$F_{1}=F_{2}=F_{3}$,

$\sigma_{1} A_{1}=\sigma_{2} A_{2}=\sigma_{3} A_{3}$,

$\sigma_{1} t_{1}=\sigma_{2} t_{2}=\sigma_{3} t_{3}$

$K_{1} \varepsilon_{1}^{n_{1}} t_{1}=K_{2} \varepsilon_{2}^{n_{2}} t_{2}=K_{3} \varepsilon_{3}^{n_{3}} t_{3}$,

$\varepsilon_{i}=\ln \left(L_{i}^{\prime} / L_{i}\right)$.

In these equations, $F_{i}$ is the tension force, $\sigma_{i}$ is the tension stress, $K_{i}$ is the strengthening coefficient, $\varepsilon_{i}$ is the true strain, $n_{i}$ is the hardening exponent, $t_{i}$ is the blank thickness, $L_{i}$ is the initial length of blank, $L_{1}^{\prime}$ is the length of blank after deformation, and $A_{i}$ is the blank section area perpendicular to the tension direction. The subscripts $i=1,2,3$, respectively, represent the thinner side, the thicker side and the thickness transition zone.

As demonstrated by Eq. (3), the stress on the thicker side and the stress on the thinner side are inversely proportional to the blank thickness. That is to say, the more the thickness gap between the thicker side and the thinner side is, the greater the stress on the thinner side is than that on the thicker side. Thus, the stress concentration on the thinner side accelerates the occurrence of the necking phenomenon.

After TRB is annealed, supposed that $K_{1}=K_{2}=K_{3}=K, n_{1}=n_{2}=n_{3}=n$, and then, it can be derived from Eq. (4) that:

$\frac{\varepsilon_{1}}{\varepsilon_{2}}=\exp \left(\frac{1}{n} \ln \frac{t_{2}}{t_{1}}\right)$.

As demonstrated by Eq. (6), the increasing thickness ratio of the blank will cause the increasing strain ratio of the thinner side to the thicker side, which may induce the earlier crack on the thinner side. 


\subsection{Derivation of Deformation Formulae}

From above analysis, it is known that for the uniaxial tension specimen of TRB, the necking phenomenon usually occurs on the thinner side. When the necking phenomenon happens, based on the definition of strain $\varepsilon=\ln \left(L^{\prime} /\right.$ $L)=\ln (1+\Delta L / L)$ and exponential constitutive relation $\sigma=K \varepsilon^{n}$, the calculation formula of deformation $\Delta L_{1}$ on the thinner side can be obtained:

$\sigma_{\mathrm{b}}\left(1+\frac{\Delta L_{1}}{L_{1}}\right)=K_{1}\left[\ln \left(1+\frac{\Delta L_{1}}{L_{1}}\right)\right]^{n_{1}}$.

According to Formula (2) and the constancy of volume, when the stress on the thinner side amounts to the necking stress $\sigma_{\mathrm{b}}$, the stress on the thicker side is expressed as:

$\sigma_{2}=\frac{\sigma_{\mathrm{b}} A_{1}^{\prime}}{A_{2}^{\prime}}=\frac{\sigma_{\mathrm{b}} \frac{A_{1} L_{1}}{L_{1}}}{\frac{A_{2} L_{2}}{L_{2}}}=\frac{\sigma_{\mathrm{b}} \frac{B_{1} L_{1} L_{1}}{L_{1}}}{\frac{B_{1} L_{2}}{L_{2}}}=\sigma_{\mathrm{b}} \frac{t_{1}}{t_{2}} \frac{L_{1}}{L_{2}} \frac{L_{2}^{\prime}}{L_{1}^{\prime}}$.

Based on $\sigma=K \varepsilon^{n}$, the calculation formula of deformation $\Delta L_{2}$ on the thicker side can be obtained:

$\sigma_{\mathrm{b}}\left(1+\frac{\Delta L_{2}}{L_{2}}\right) \frac{t_{1}}{t_{2}} \frac{L_{1}}{L_{2}} \frac{\Delta L_{2}+L_{2}}{\Delta L_{1}+L_{1}}=K_{2}\left[\ln \left(1+\frac{\Delta L_{2}}{L_{2}}\right)\right]^{n_{2}}$.

In the above equations, $\sigma_{\mathrm{b}}$ is the necking stress on the thinner side, $\Delta L_{i}$ is the blank deformation $(i=1,2)$, and $A_{i}^{\prime}$ is the blank section area after deformation $(i=1,2)$.

Therefore, in the circumstances that the material parameters of TRB as well as the necking stress on the thinner side are known, Eq. (7) is used to solve the deformation on the thinner side of TRB. Then, the deformation on the thicker side can be worked out by Eq. (9).

\section{Uniaxial Tension Test and Simulation on TRB}

\subsection{Uniaxial Tension Test}

At present, there is no definite international standard on the shape of TRB specimen for uniaxial tension, and ASTM standard is adopted for all the tests in this paper. A resistive extensometer is used to measure the deformation of TRB, which has a gauge length of $50 \mathrm{~mm}$ and a maximum scale value of $25 \mathrm{~mm}$. The elongations on the thinner side and on the thicker side are, respectively, measured by a vernier caliper with the precision of $0.01 \mathrm{~mm}$. Figure 2 presents the tension specimen of TRB, and $1.2 \mathrm{~mm}$ thinner blank and $2.0 \mathrm{~mm}$ thicker blank also have the same dimension as shown in Fig. 2. The investigated material is SPHC steel, and its chemical composition (wt\%) is C 0.083 , Si 0.041, Mn 0.316, S 0.012, P 0.017 and balanced of Fe. Specimens of $1.2 / 2.0 \mathrm{~mm}$ TRB are cut out in the rolling direction $\left(0^{\circ}\right)$

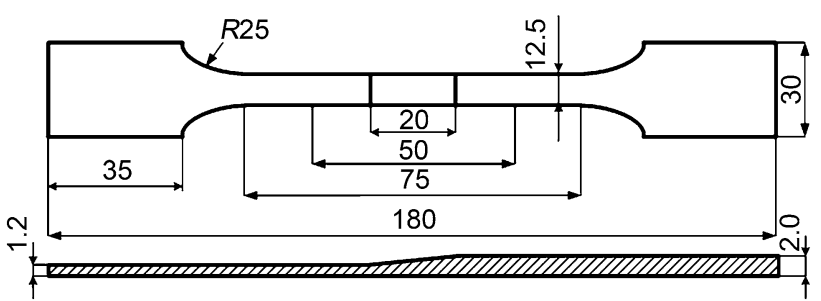

Fig. 2 Dimension of tension specimen for TRB (mm)

of SPHC sheet with variable thickness, and meanwhile, $1.2 \mathrm{~mm}$ thinner blank and $2.0 \mathrm{~mm}$ thicker blank are cut out, respectively, in the direction of $0^{\circ}, 45^{\circ}$ and $90^{\circ}$ [13]. Some of the specimens are annealed, and the annealing process route is shown in Fig. 3 .

Performance parameters for the thinner side and the thicker side of TRB obtained by uniaxial tension test are given in Tables 1 and 2 . With the related parameters and the dimension of the specimen substituted in Eqs. (7) and (9), the deformations on the thinner side and on the thicker side of TRB can be obtained, respectively. Comparison of deformation results obtained through analysis and test is given in Table 3. It is observed that the analytical values match the test values well. And it is also proven that the nonuniform deformation formulae for the thinner side and the thicker side are accurate. In addition, whether TRB is annealed or not, the deformation on the thinner side is much larger than that on the thicker side, and the deformation mainly happens on the thinner side.

According to Table 3, although the analytical values are close to the experimental values, there is still an error between them. The error occurs mainly during the course of measurement. The transition zone moves during deformation, and the measuring base lines (brinks of the transition zone) will also move, which will increase the difficulty of measurement and bring a larger error. Besides, when the elongations on the thinner side and on the thicker side were, respectively, measured by a vernier caliper,

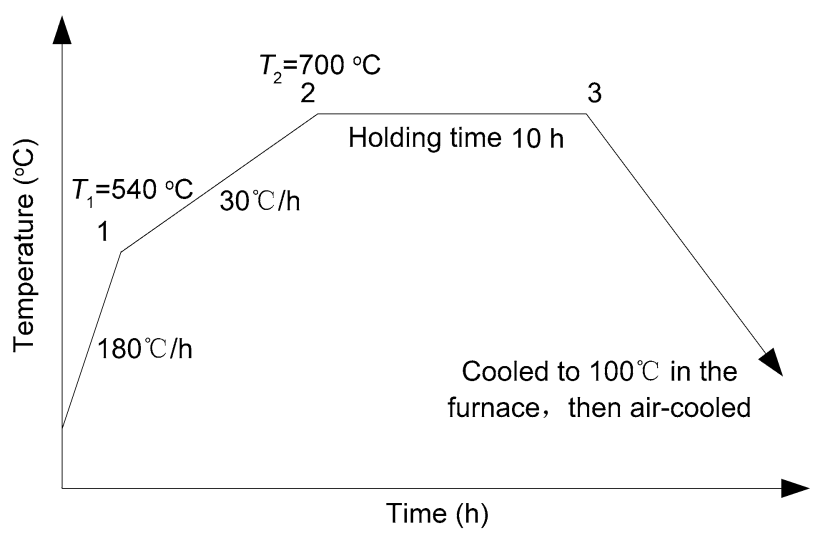

Fig. 3 Schematic diagram of two-slope annealing process 
Table 1 Mechanical properties of unannealed TRBs with thicknesses of 1.2 and $2.0 \mathrm{~mm}$

\begin{tabular}{llllllrr}
\hline Thickness $(\mathrm{mm})$ & $E(\mathrm{GPa})$ & $\sigma_{\mathrm{s}}(\mathrm{MPa})$ & $\sigma_{\mathrm{b}}(\mathrm{MPa})$ & $n$ & $r$ & $K(\mathrm{MPa})$ & $\delta(\%)$ \\
\hline 1.2 & 179 & 209.6 & 300.9 & 0.24 & 1.56 & 533.9 & 35.2 \\
2.0 & 195 & 209.4 & 271.7 & 0.22 & 1.09 & 459.8 & 38.2 \\
\hline
\end{tabular}

Table 2 Mechanical properties of annealed TRBs with thicknesses of 1.2 and $2.0 \mathrm{~mm}$

\begin{tabular}{llllllrr}
\hline Thickness $(\mathrm{mm})$ & $E(\mathrm{GPa})$ & $\sigma_{\mathrm{s}}(\mathrm{MPa})$ & $\sigma_{\mathrm{b}}(\mathrm{MPa})$ & $n$ & $r$ & $K(\mathrm{MPa})$ & $\delta(\%)$ \\
\hline 1.2 & 186 & 170.7 & 237.5 & 0.27 & 1.98 & 443 & 42.5 \\
2.0 & 196 & 179.5 & 264.9 & 0.24 & 1.50 & 472.5 \\
\hline
\end{tabular}

Table 3 Comparisons of deformation between analytical result and experimental result of unannealed and annealed TRBs

\begin{tabular}{llllll}
\hline $\begin{array}{l}\text { TRB } \\
\text { sample }\end{array}$ & \multicolumn{2}{l}{ Thinner } & & & \multicolumn{2}{l}{ Thicker } \\
\cline { 2 - 3 } \cline { 5 - 6 } \cline { 5 - 6 } & Analytical & Experimental & & Analytical & Experimental \\
\hline Unannealed & 10.65 & 11.21 & 0.23 & 0.42 \\
Annealed & 12.27 & 14.66 & & 0.16 & 0.35 \\
\hline
\end{tabular}

because of the precision of the vernier caliper and the width of the base lines for measurement, an error is inevitable. The vernier caliper used in this paper has the precision of $0.01 \mathrm{~mm}$, and meanwhile, the elongation on the thicker side is very small, so the relative error for the thicker side is larger.

Figure 4 shows the unannealed and annealed TRB specimens after test. It can be seen that, after annealing, there is much improvement in terms of blank elongation rate. Moreover, necking failure happens on the thinner side, which agrees with the discussions on Eqs. (7) and (9). The reason is that annealing can release the residual stress in the rolling process, which decreases the strength and improves the plasticity in TRB [14].

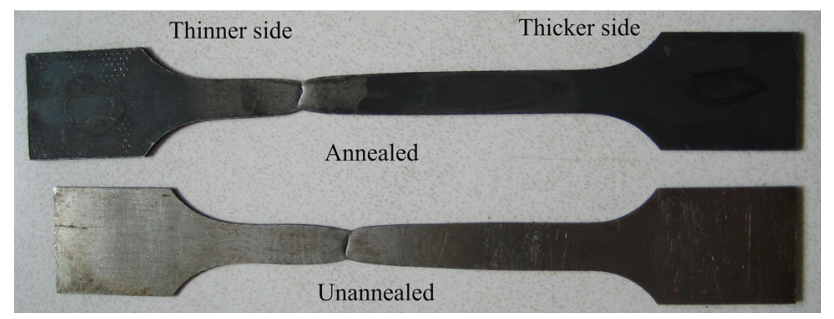

Fig. 4 Comparison between unannealed and annealed TRB specimens after test

\subsection{Construction of Stress-Strain Field}

Lagrange interpolation polynomial can be expressed as:

$p_{n}(x)=\sum_{i=0}^{n} a_{i} l_{i}(x)$.

According to the interpolation polynomial principle and the nature of Lagrange primary function:

$p_{n}\left(x_{i}\right)=f\left(x_{i}\right)=\sum_{j=0}^{n} a_{j} l_{j}\left(x_{i}\right)=a_{i}, \quad i=0,1, \ldots, n$.

So Lagrange interpolation polynomial can be rewritten as:

$p_{n}(x)=\sum_{i=0}^{n} f\left(x_{i}\right) l_{i}(x)$.

Where Lagrange primary function is:

$l_{i}(x)=\frac{\left(x-x_{0}\right)\left(x-x_{1}\right) \cdots\left(x-x_{i-1}\right)\left(x-x_{i+1}\right) \cdots\left(x-x_{n}\right)}{\left(x_{i}-x_{0}\right)\left(x_{i}-x_{1}\right) \cdots\left(x_{i}-x_{i-1}\right)\left(x_{i}-x_{i+1}\right) \cdots\left(x_{i}-x_{n}\right)}$
$i=0,1, \ldots, n$.

$l_{i}(x)$ is a polynomial of degree $n$ and has the following nature:

$l_{i}\left(x_{j}\right)=\delta_{i j}=\left\{\begin{array}{l}1, i=j \\ 0, i \neq j\end{array} \quad i, j=0,1, \ldots, n\right.$.

$l_{0}(x), l_{1}(x), \ldots, l_{n}(x)$ are linearly independent quantities and are Lagrange primary functions of $x_{0}, x_{1}, \ldots, x_{n}$.

Lagrange polynomial interpolation method above is used to construct the stress-strain field by taking the blank thickness as variable in order to obtain material parameters of TRB, based on test data of 1.2 and $2.0 \mathrm{~mm}$ blanks with invariable thickness from Tables 2 and 3, which can 
provide reliable mechanical parameters of TRB for numerical simulation, as shown in Fig. 5.

For the uniform thickness sides of TRB, related parameters can be directly obtained from Tables 2 and 3 . For the thickness transition zone of TRB, finite element method can be applied to scatter it into finite number of combined blanks. Then, the scattered blanks are put together to conduct calculation of the whole transition zone, as shown in Fig. 6. The more precisely the blank is divided, the closer the property of the combination is to that of the thickness transition zone. The continuous thickness change of the transition zone corresponds to the material parameters of different thicknesses in the true stress-strain field in Fig. 5. Thus, through the stress-strain field, mechanical parameters of various thicknesses for the transition zone are obtained. Therefore, TRB modeling in numerical simulation is settled with finite element method.

\subsection{Uniaxial Tension Simulation}

Figure 7 shows the finite element model of uniaxial tension on $1.2 / 2.0 \mathrm{~mm}$ TRB. The model is meshed by means of eight-node solid element. The material parameters obtained from the stress-strain fields in Fig. 5 are assigned to the elements with corresponding thicknesses. The material is subject to von Mises yield criterion and exponential hardening method. In the process of simulation, all the degrees of freedom for the clasp side on the thinner side are restricted, and the displacements obtained from the tension tests are applied as boundary condition to be imposed on the clasp side on the thicker side [12].

Figure 8 shows the equivalent effective strain distribution of unannealed and annealed uniaxial tension specimens of 1.2/2.0 mm TRB when the necking happens.

Figure 8 demonstrates that the deformations of unannealed and annealed TRB specimens concentrate on the thinner sides until the necking failure happens. In the case of equivalent displacement, the annealed specimen has the smaller equivalent effective strain and thus can obtain

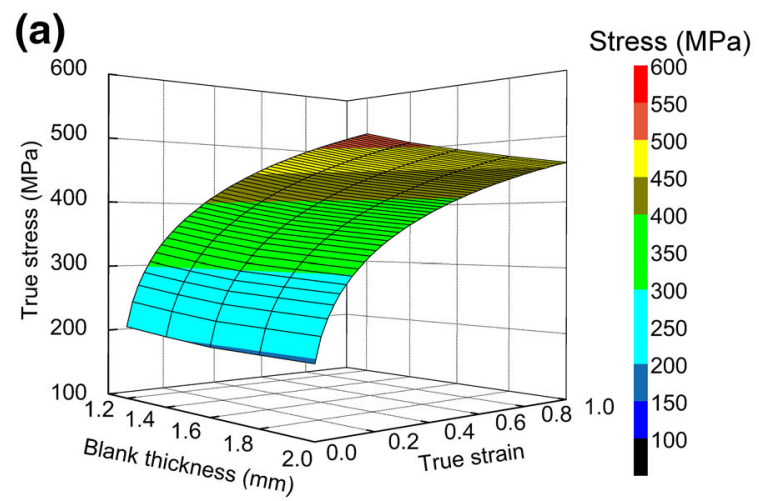

Fig. 5 True stress-strain fields of TRBs: a unannealed, b annealed

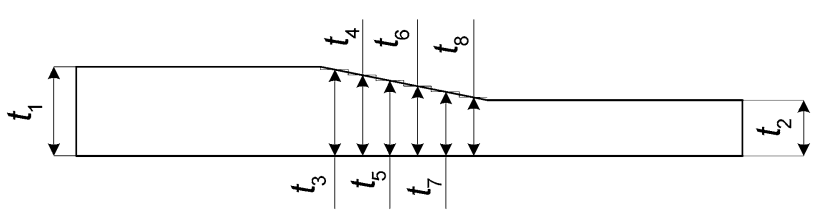

Fig. 6 Discretization of TRB transition zone

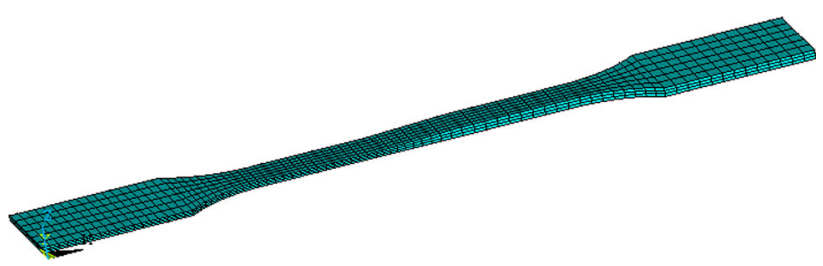

Fig. 7 Finite element model of uniaxial tension for TRB in simulation

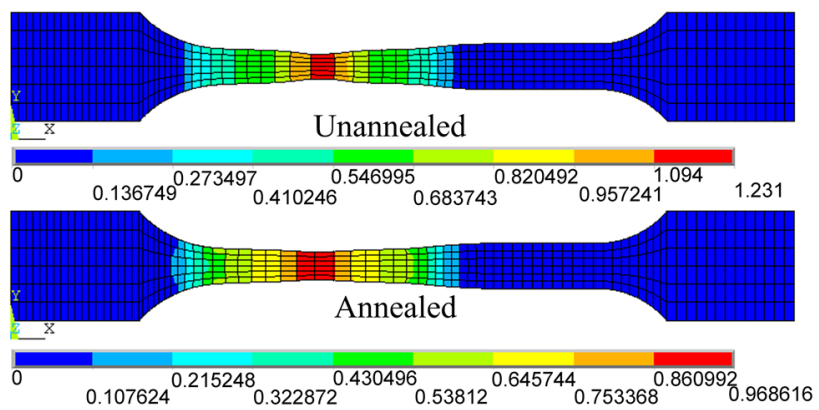

Fig. 8 Equivalent strain distribution of TRB specimen when necking happens

much greater elongation rate. Simulation results are in line with discussions on Eqs. (7) and (9), and they also match the test results.

Figure 9 shows the comparison of displacement-load curves between TRB uniaxial tension test and simulation. From Fig. 9, it is observed that the annealed TRB gets greater elongation rate, and the displacement-load curves calculated by simulation greatly match the test curves

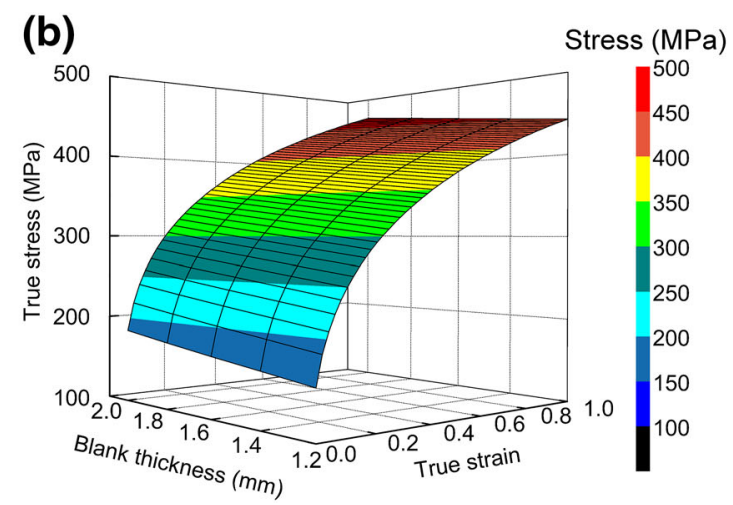




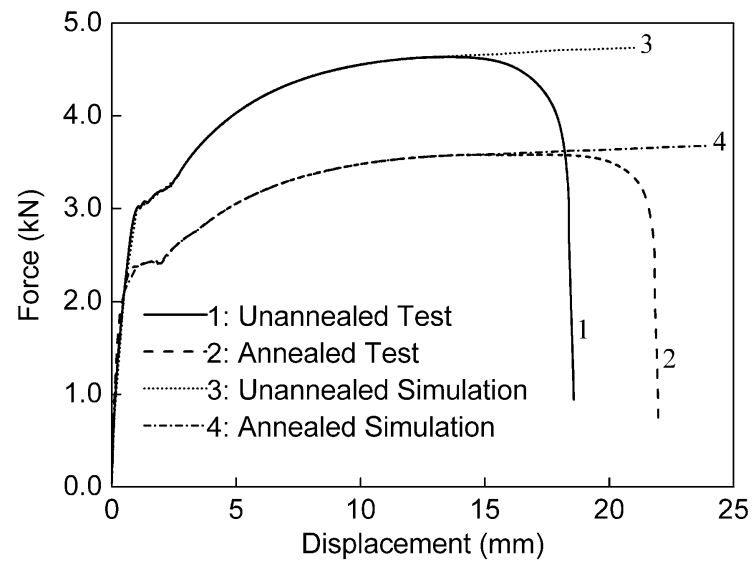

Fig. 9 Comparison of displacement-load curves of TRBs between tension test and simulation

before necking. However, the necking in simulation occurs later than that in test does. The reason is that, in the test, TRB will crack where the necking appears, and the stressstrain curves used in simulation are obtained by interpolating the test data, whereas the stress-strain curves after necking are obtained by extrapolation.

\section{Metallurgical Structure}

Microstructures were observed under a metallurgical microscope and are shown in Fig. 10. It can be seen from Fig. 10a-c that grains in the transition zone are elongated in the rolling direction, compared with those on the $2.0 \mathrm{~mm}$ thicker side. Meanwhile, on the thinner side, ferritic grains are deformed more obviously. Partial tissues show fibrous structures in the rolling direction, and the sizes of grains are not uniform. Therefore, the strength of the unannealed TRB increases and the plasticity decreases. The strength of the thinner side is higher than that of the thicker side, and the plasticity of the thinner side is lower than that of the thicker side, so crack happens on the thinner side.

According to Fig. 10d-f, massive uniform equiaxed grains and pie-shaped grains exist in the microstructure, so the strength decreases and the plasticity increases. Some grains become to grow, and the pie-shape degree increases. All of above is advantageous to acquiring excellent formability $[15,16]$, so the blank elongation rate increases, and the whole TRB sheet shows more uniform properties. Annealing releases the residual stress produced in the rolling process, which decreases the strength and improves the plasticity in TRB, especially on the thinner side. Nevertheless, the strength and the plasticity of the thinner side are both smaller than those of the thicker side [17], so the deformation concentrates on the thinner side, and the crack occurs on the thinner side.

\section{Conclusions}

In the process of uniaxial tension for TRB, nonuniform deformation will happen, and the necking phenomenon for uniaxial tension specimens happens on the thinner side. The mechanical analytical model of TRB can accurately
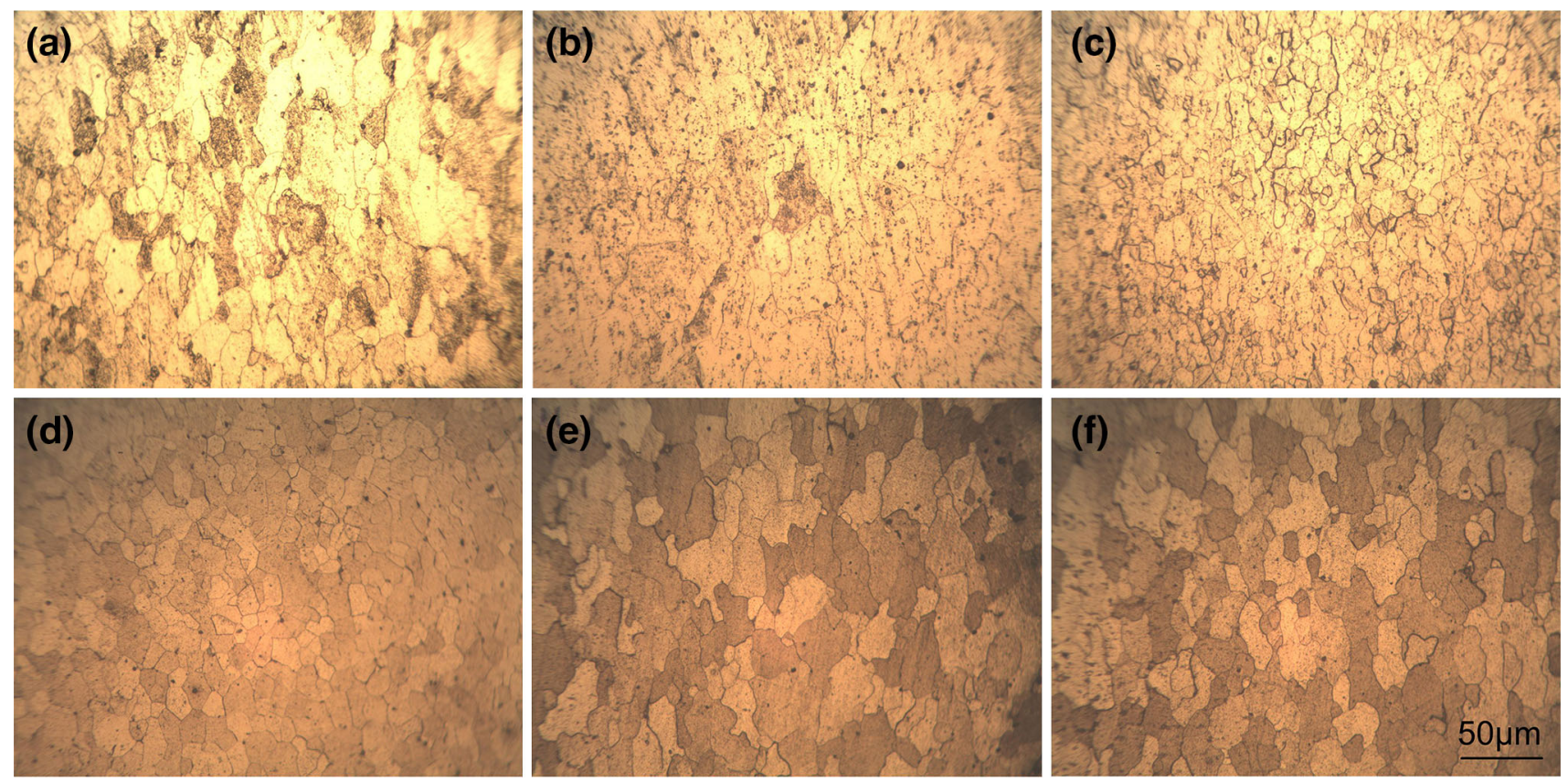

Fig. 10 Microstructures of unannealed and annealed TRBs: a unannealed $1.2 \mathrm{~mm}$ side, $\mathbf{b}$ unannealed transition zone, $\mathbf{c}$ unannealed $2.0 \mathrm{~mm}$ side, d annealed $1.2 \mathrm{~mm}$ side, $\mathbf{e}$ annealed transition zone, $\mathbf{f}$ annealed $2.0 \mathrm{~mm}$ side 
describe the deformation process of TRB during uniaxial tension. The deformation of TRB can be quantified by the nonuniform deformation formulae for the thinner side and the thicker side. The construction of stress-strain fields combined with finite element method can solve TRB modeling in numerical simulation. Analysis, test and simulation results have great consistency, which also verifies the accuracy of the model and the formulae. The research results could be well explained by metallurgical structure.

Acknowledgments This work was financially supported by the National Natural Science Foundation of China (Nos. 51105068 and 51475086), the Fundamental Research Funds for the Central Universities (Nos. N130323003 and XNB201413) and the Science and Technology Research Project for Higher School of Hebei Province (No. Z2013068).

\section{References}

[1] X.H. Liu, Q.L. Zhao, L.Z. Liu, Acta Metall. Sin. (Engl. Lett.) 27, 483 (2014)

[2] R.N. Ganesh, B.S. Naik, Mater. Manuf. Process. 25, 1351 (2010)

[3] A. Meyer, B. Wietbrock, G. Hirt, Int. J. Mach. Tool. Manuf. 48, $522(2008)$
[4] M. Merklein, M. Johannes, M. Lechner, A. Kuppert, J. Mater. Process. Technol. 214, 151 (2014)

[5] S. Xue, J. Zhou, Y.Q. He, Forg. Stamp. Technol. 36, 30 (2011). (in Chinese)

[6] X.H. Liu, J. Iron Steel Res. Int. 18, 1 (2011)

[7] Y.J. Wu, J.P. Lin, Q.S. Hu, R.Q. Guo, W.H. Bao, J. Plast. Eng. 16, 35 (2009). (in Chinese)

[8] J.P. Lin, D.Y. Xing, Q.S. Hu, S.S. Chen, J. Tongji Univ. Nat. Sci. 38, 1350 (2010). (in Chinese)

[9] S.S. Chen, J.P. Lin, L.J. Xin, Mater. Sci. Technol. 18, 480 (2010). (in Chinese)

[10] S.S. Chen, J.P. Lin, J. Mater. Eng. (10), 33 (2011) (in Chinese)

[11] Y.H. Li, J.T. Du, H.B. Fei, Mod. Manuf. Eng. (3), 83 (2010) (in Chinese)

[12] X.J. Bao, Dissertation, Shanghai Jiaotong University, Shanghai, 2003 (in Chinese)

[13] X.R. Deng, G.J. Zhang, X.H. Liu, Forg. Stamp. Technol. 39, 32 (2014). (in Chinese)

[14] H.W. Kim, C.Y. Lim, Mater. Des. 31, 71 (2010)

[15] L.F. Qiao, Z.Y. Liu, X.H. Liu, G.D. Wang, J. Iron Steel Res. 23, 43 (2011). (in Chinese)

[16] B.H. Zheng, L.J. Wang, J.Y. Lu, D.W. Lv, Y.L. Kang, Steel Roll. 23, 4 (2006). (in Chinese)

[17] Y. Zhi, Y. Tian, J.L. Zhang, X.H. Liu, J. Northeast. Univ. Nat. Sci. 35, 671 (2014). (in Chinese) 